\title{
Optimization of the Parameters in the RHIC Single Crystal Heavy Ion Collimation
}

\author{
V.M.Biryukov, Yu.A.Chesnokov, V.I.Kotov \\ IHEP, Protvino, Russia \\ D. Trbojevic, A. Stevens \\ BNL, Upton, USA \\ Presented at the 1999 Particle Accelerator Conference, New York
}

\begin{abstract}
In the framework of the project to design and test a collimation system prototype using bent channeling crystal for cleaning of the RHIC heavy ion beam halo, we have studied the optimal length and bending angle of a silicon (110) single crystal proposed to be a primary element situated upstream of the traditional heavy amorphous collimator. Besides the matters of the channeling and collimation efficiency, we also looked into the impact the crystal may have on the non-channeled particles that go on circulating in the ring, so as to reduce the momentum offset of the particles scattered of the crystal.
\end{abstract}

\section{INTRODUCTION}

The project of a collimation system using bent channeling crystal for cleaning of the RHIC heavy ion beam halo has been described in some detail in Ref.[1] where we refer for the basic ideas and results, and more technical information. In order to optimize the crystal collimation scheme for RHIC, we have studied the influence of the silicon (110) single crystal parameters (length, bending angle, curvature gradient) on the efficiency of bending of the beam of fully ionized Au ions. The beam distribution at the entrance of the crystal was presented by the sample of 19258 fully stripped gold ions generated as described in our previous article [1].

We have also postulated that at the entrance to the crystal all the particles have the same momenta, corresponding to the Lorentz factor of 108.40, in order to evaluate then the momentum distribution in the beam downstream of the crystal.

In our first study [1] the crystal length of $10 \mathrm{~mm}$ and the bending angle of $1 \mathrm{mrad}$ were used, as it was a practical choice corresponding to the state of art on that date. It was concluded that the bending angle can be reduced substantially. In the mean time, the IHEP Protvino experimental practice has shown that shorter crystals of high quality can be produced and they behave in perfect agreement with theory [2]. 


\section{TRACKING IN CRYSTAL}

With the above said arrangements, we have performed tracking of the 19258 particles through various crystals by means of CATCH code [3]. Typical distribution of the particles downstream of the crystal consists of a sharp peak of the particles channeled through the full crystal length and bent the full angle, some particles unbent but scattered of the crystal, few particles bent partial angle between the bent and unbent peaks, and few particles nuclear interacted in the crystal. Fig. 1 represents gold ion distribution in the horizontal phase space at the downstream edge of the crystal.

Having studied such distributions for the crystals with different length but the same bending angle of $0.5 \mathrm{mrad}$, we plot in Fig. 2 how many particles were bent at least $0.1 \mathrm{mrad}$ (this includes the particles channeled part of the crystal length as they are steered through the angles that might be sufficient for interception by the downstream collimator). One can see that, from standpoint of physics alone, one can reduce the crystal length to nearly $2 \mathrm{~mm}$ without seriously affecting the efficiency of bending of the above-defined sample of particles.

Further we studied how the efficiency of the 5-mm long crystal would depend on the bending angle in the range of about $0.2-1.0 \mathrm{mrad}$. There was hardly any dependence up to at least $1 \mathrm{mrad}$, so this gives us the freedom to adjust the bending angle to what seems appropriate for collimation purpose in RHIC.

One objective on the agenda was to find if we could benefit in the efficiency from a variable curvature of the crystal (in the above simulations the curvature was constant). The motive is that, whereas in the extraction mode one has to deliver the bent beam to a single direction, in the collimation mode it is sufficient to deliver the bent beam to a range of directions, say from 0.3 to $1.0 \mathrm{mrad}$ in our case, as the particles are absorbed anyway (it might be even useful to spread the irradiation load over the collimator). In the simulation we have tried variable curvature of the 5-mm crystal, with the curvature peak being factor of 2, 4, 8, and 16 of the average. However, the fraction of the particles bent at the angles greater than $0.1 \mathrm{mrad}$ has not changed significantly as compared to the above studies, so we don't mention this option further on.

Table 1 summarizes comparative characteristics of the beam interaction with two crystals, $5 \mathrm{~mm}$ long and $10 \mathrm{~mm}$ long. The total number of the gold ions incident on the crystals was $N$ particles. The number of particles which interacted with the crystal nuclei was $N_{\text {Lostparticles }}$. The $N_{\text {channeled }}$ represents the number of particles channeled through the full length of the crystal and hence bent the full angle, and Eff is their fraction to the total.

The momentum distribution has substantially improved with shorter crystals as compared to the earlier study [1]. The $N_{\text {inbucket }}$ and $N_{\text {outoffbucket }}$ in Table 1 show the corresponding number of the particles from the momentum distribution downstream of the crystal. Figure. 3 shows phase space of the gold ions at the end of the 5-mm long crystal, where on the $\mathrm{x}$ coordinate is shown as $\mathrm{dp} / \mathrm{p}$ dependent. Taking into account also the practical considerations and experience obtained with crystals at IHEP Protvino, we can conclude that 5-mm long crystal seems well suited for the job of Au beam bending at the angle of the order of $0.5 \mathrm{mrad}$ in a single passage. Several crystals of this size have been already produced and tested at Protvino [2].

One option for the future simulation studies may sound interesting. Fig. 2 shows 
Table 1: The results of beam interaction with two Si(110) crystals.

\begin{tabular}{|c|c|}
\hline Crystal 1 cm & Crystal $0.5 \mathrm{~cm}$ \\
\hline 19258 & 19258 \\
962 & 456 \\
12532 & 13686 \\
17289 & 18628 \\
467 & 174 \\
$65.1 \%$ & $71.1 \%$ \\
\hline
\end{tabular}

that from physics considerations we can reduce the crystal length to order of 1-2 mm, if it appears convenient technically. Such crystal would make a rather little disturbance to the circulating beam, thus allowing potentially many passages of the circulating particles through the bent crystal and enhancing the overall efficiency of bending. To benefit from this mode, such a crystal should have also a sufficient transversal size (possibly greater than the longitudinal size). Studies on feasibility of such crystal deflectors are in progress.

\section{COLLIMATION SIMULATION IN RHIC}

The gold ions tracked through the crystal have been transported through the RHIC ring using the tracking program TEAPOT [4], with accelerator setting as described in Ref. [1]. Fig. 4 shows the losses around the RHIC rings from the particles scattered of the primary and secondary collimators and the losses from the particles deflected by the crystal. Two extreme cases are presented when the primary collimator downstream of the crystal is wide open and when it is set at $5 \sigma_{x}$, the same horizontal distance as a front edge of the crystal.

\section{CONCLUSIONS}

An improvement in the momentum distribution of the ions passed through the crystal and a reduction of lost particles around the ring have been achieved by optimizing the crystal length. Another result is the freedom we have in choosing the angle of bending, which can be left about $0.5 \mathrm{mrad}$, or be chosen from consideration of collimation physics, or be just varied in the course of experimentation at RHIC.

\section{References}

[1] D. Trbojevic et al., "A Study Of RHIC Crystal Collimation," arXiv:hepex/0111021] in EPAC Proceedings (Stockholm, 1998) and references therein. V. Biryukov, Proceedings of the "International Symposium on Near Beam Physics", 
at Fermi National Laboratory, Batavia, Illinois, USA, Sptember 22-24, 1997, pp. 179-184 arXiv:hep-ex/0110074].

[2] A. G. Afonin et al., JETP Lett. 68, 568 (1998) Phys. Lett. B 435, 240 (1998). JETP Lett. 67, 781 (1998) arXiv:hep-ex/0111028.

[3] Valery Biryukov, "Crystal Channeling Simulation - CATCH 1.4 User's Guide", SL/Note 93-74(AP), CERN, 1993. V. Biryukov, Phys. Rev. E 51, 3522 (1995). Phys. Rev. E 52, 2045 (1995). Phys. Rev. E 52, 6818 (1995). Phys. Rev. Lett. 74, 2471 (1995).

[4] L.Schachinger and R.Talman, "A Thin Element Accelerator Program for Optics and Tracking", SSC Central Design Group, Internal Report SSC-52 (1985). 


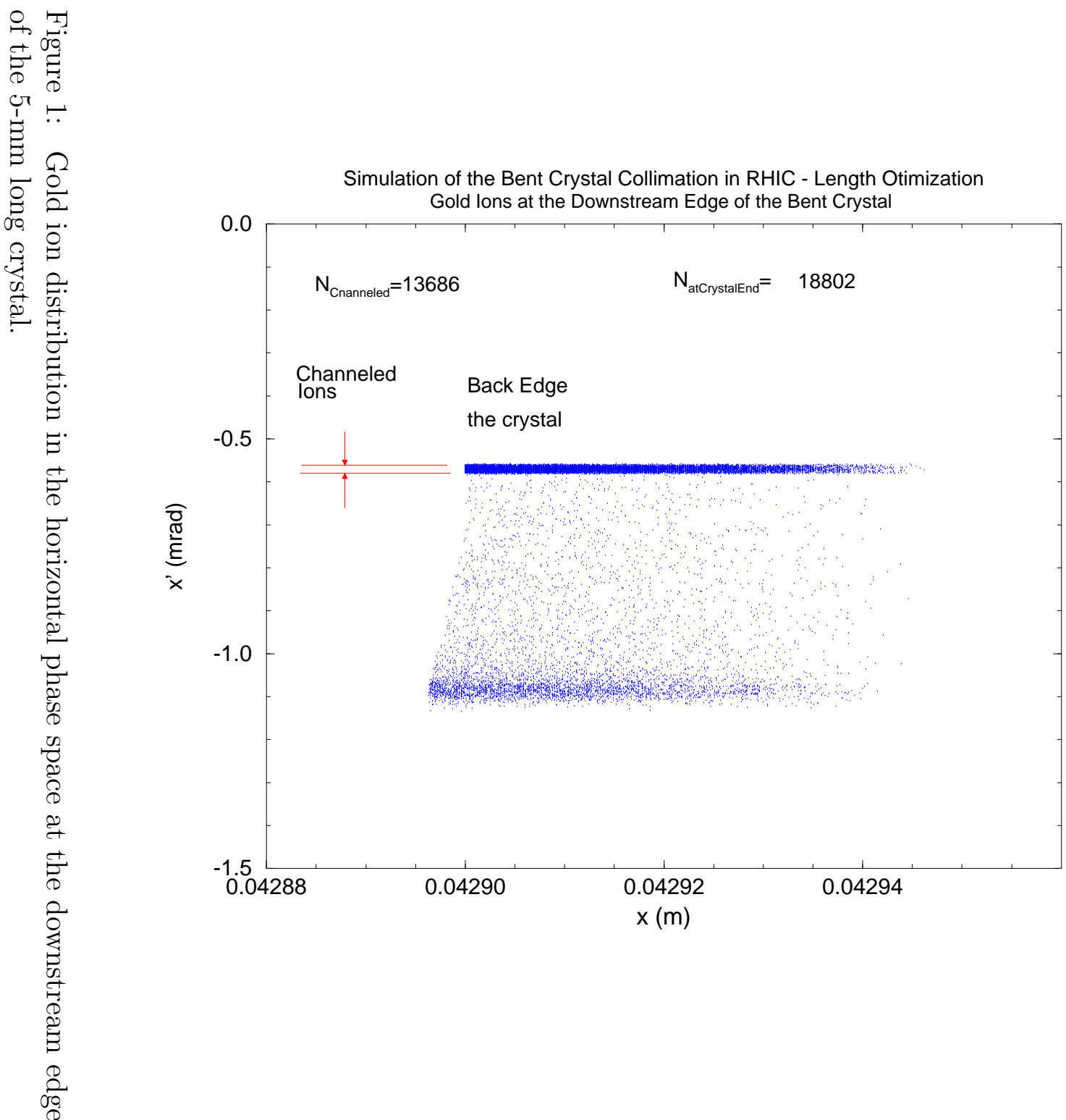




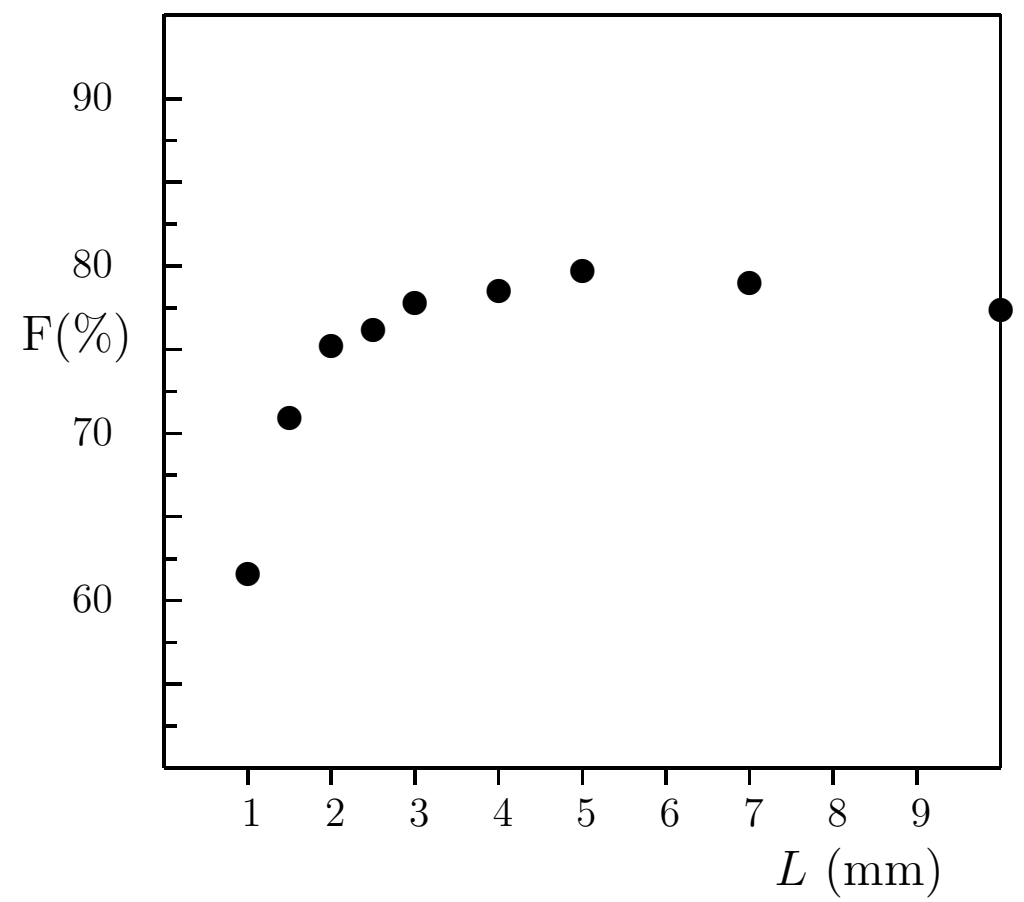

Figure 2: Single-pass bending efficiency vs crystal length; for $0.5 \mathrm{mrad}$ bending angle. 


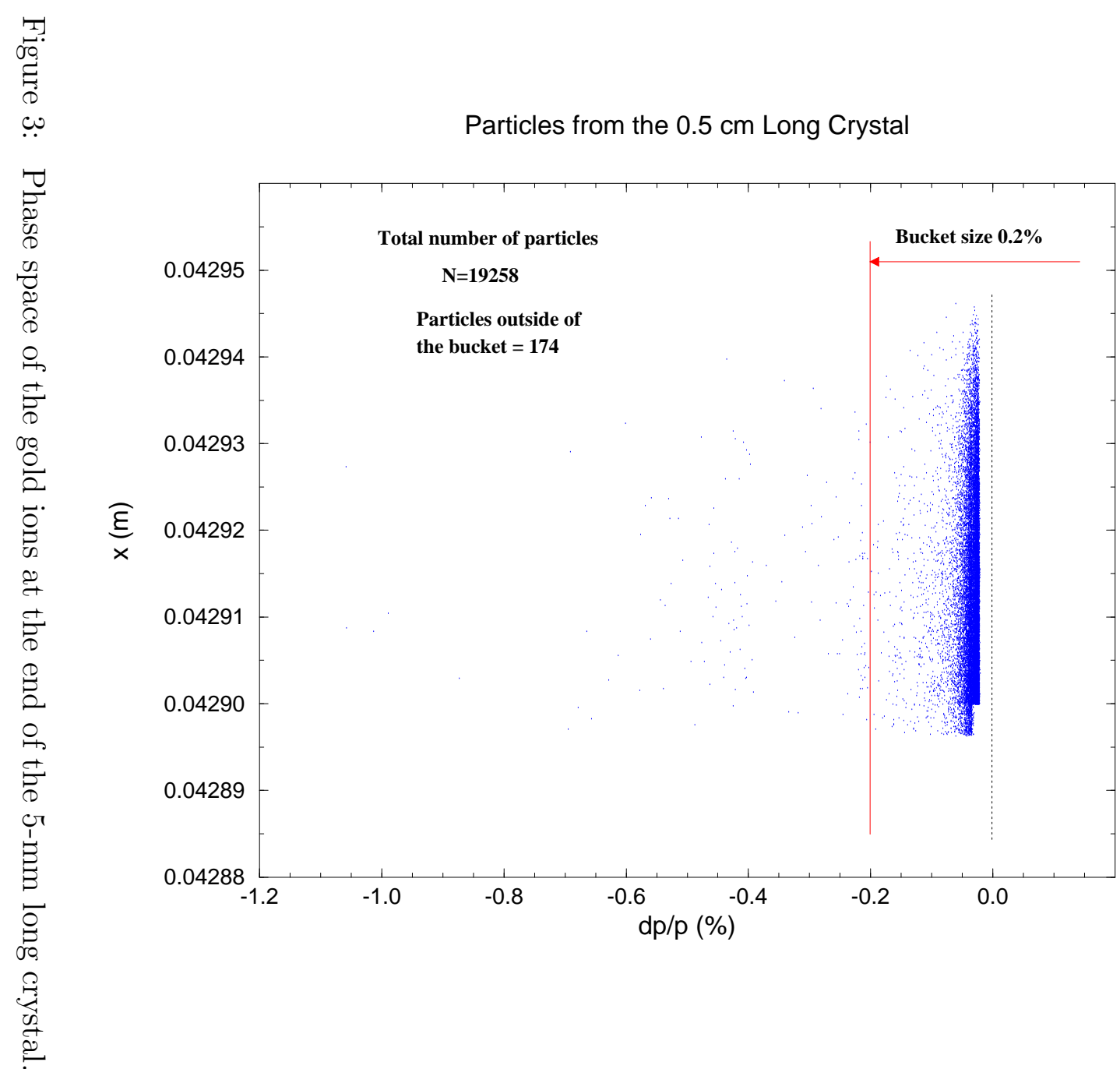




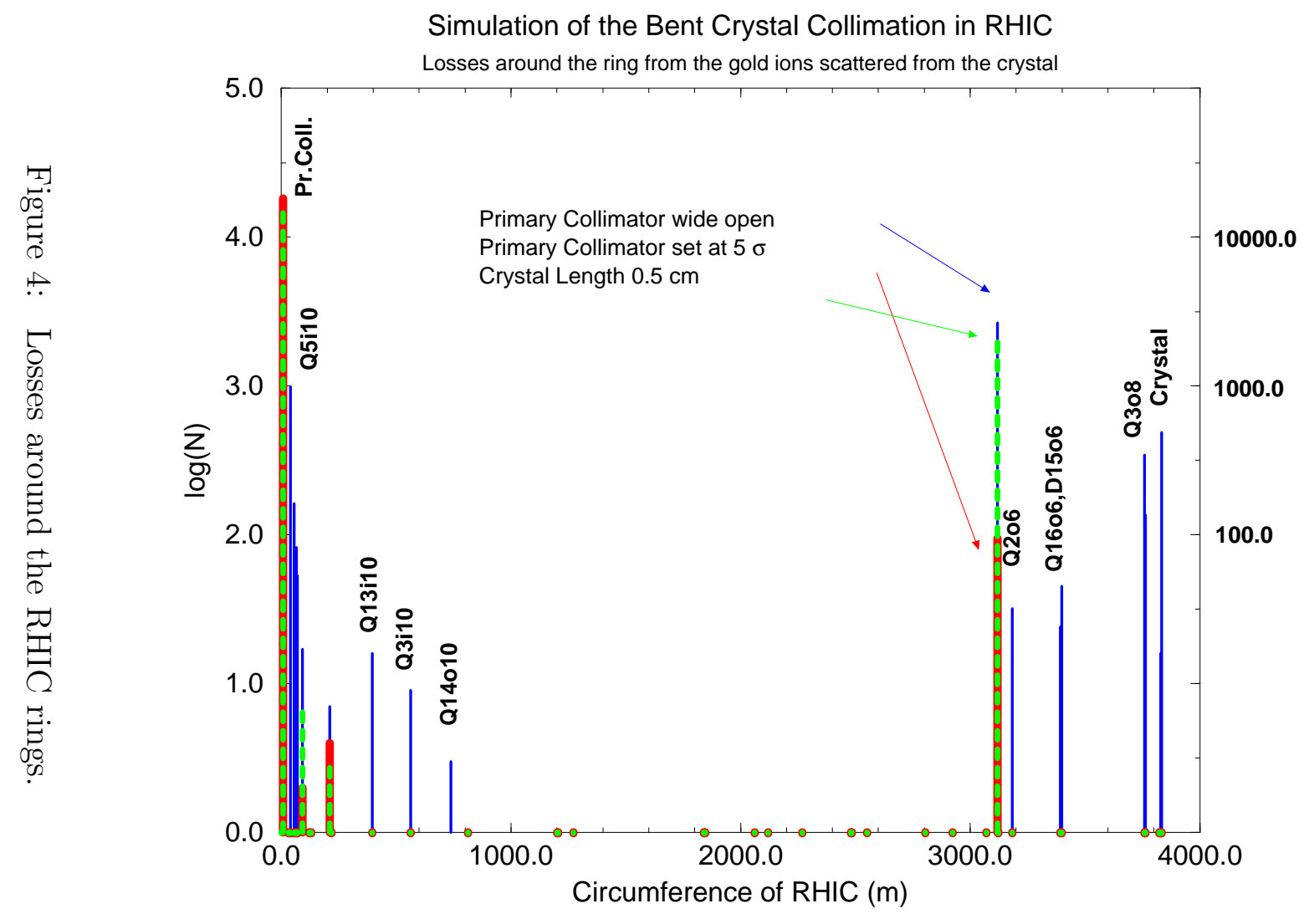

